\title{
Fatigue Damage Analysis of an Asphalt Mixture Based on Pseudostiffness
}

\author{
X. R. Wang, ${ }^{a}$ B. Y. Yin, ${ }^{a}$ and W. B. Luo ${ }^{\mathrm{a}, \mathrm{b}, 1}$ \\ ${ }^{a}$ College of Civil Engineering and Mechanics, Xiangtan University, Xiangtan, China \\ b Hunan Key Laboratory of Geomechanics and Engineering Safety, Xiangtan University, Xiangtan, \\ China \\ ${ }^{1}$ luowenbo@xtu.edu.cn
}

The fatigue damage characteristics of an asphalt mixture were analyzed, a new fatigue damage model was constructed based on the Schapery pseudovariable theory and continuum damage mechanics. According to the Schapery theory and the generalized elastic-viscoelastic correspondence principle, the experimental stress-strain curves of the asphalt mixture are transformed into the stress vs. pseudostrain curves. The relation between stress and pseudostrain is shown to be linear, which eliminates the viscoelastic hysteresis effect of the asphalt mixture and its time dependence. Pseudostiffness decreases gradually with the number of fatigue cycles. The damage variable is defined by the change in pseudostiffness. The parameters of the fatigue damage model are verified by fatigue tests up to 1000 cycles, and the fatigue damage for 2000 cycles is predicted and compared with the test results, which are in good agrement between themselves.

Keywords: fatigue damage, pseudostrain, fatigue lifetime, viscoelasticity, pseudostiffness.

Introduction. Asphalt pavement has excellent performance of driving comfort and noise reduction and has been used widely in road engineering. Under the cyclic action of vehicle load, the asphalt pavement will have varying degrees of damage such as cracking, raveling, potholing, and rutting [1]. The fatigue damage of asphalt mixture has been an important issue for scholars. Lee proposed a fatigue performance prediction model of asphalt concrete [2], which has been developed from a uniaxial constitutive model based on the elastic-viscoelastic correspondence principle and continuous damage mechanics by use of mathematical simplifications. Kim et al. [3] analyzed the fatigue cracking and failure of inelastic heterogeneous asphalt concrete mixtures with the finite element method and proposed a calculation model to predict the fatigue damage behavior of asphalt mixture under cyclic loading. Li et al. [4] used the fracture energy density of asphalt mixture from indirect tension test to propose a simple fatigue prediction model. Guan et al. [5] introduced the viscoelastic fatigue damage model of asphalt mixture under cyclic loading on the bases of the viscoelastic constitutive model and strain equivalent assumption. After studying the fatigue damage under cyclic loading, Zhu et al. [6] have put forward the damage model of interaction between fatigue and creep for asphalt mixture by using fatigue-creep coupling damage theory. In the above-mentioned fatigue damage models, some of these models are based on the energy dissipation principle. The others are phenomenological models relating the damage to the fatigue cycles. However, the damage variable is lack of mechanical meaning.

Developing a realistic mathematical model of the mechanical behavior of asphalt mixture with accumulation damage is complicated work. The complexity is attributed to the viscoelastic hereditary effects of the binder, the complex nature of describing the damage, the plastic and viscous flow of the binder, the friction of aggregate particles and the coupling between these mechanisms [7], However, how can these complex problems be simplified and which should be the researchers' thinking. Based on the damage mechanics, the essence of fatigue is the accumulation of material damage with the increase of cyclic loading. Thus, in this paper, the cyclic loading times in the fatigue test were contacted from 
the macro view, and according to the strain response of asphalt mixture under cyclic loading, the stress vs. pseudostrain curves was obtained. Pseudostiffness has been used to characterize the damage degree of the asphalt mixture. By use of the pseudostiffness, the mechanical properties of the asphalt mixture are related to a cyclic loading time to construct a new kind of intuitive and straightforward damage model.

1. Pseudovariable Theory. The mechanical properties of viscoelastic materials reveal time or rate dependence. The response of asphalt mixture depends not only on the current state of input but also on all history of input [8]. If the material has linear viscoelastic, its constitutive equation can be constructed by the Boltzmann superposition principle as in [9]:

$$
\sigma_{i j}=\int_{0}^{t} E_{i j k l}(t-\tau) \frac{\partial \varepsilon_{k l}}{\partial \tau} d \tau,
$$

where $\sigma_{i j}$ and $\varepsilon_{k l}$ are stress and strain, respectively, $E_{i j k l}$ is the stress relaxation function of the material, and $t$ is physical time.

By using elastic-viscoelastic correspondence principle, the viscoelastic problem can be transformed into a linear elastic problem. Schapery proposed the extended elasticviscoelastic correspondence principle which can apply to both linear and nonlinear viscoelastic materials [10]. It was suggested that the constitutive equation of viscoelastic media such as asphalt mixture was identical to that in the elastic case. Stresses and strains are not necessarily physical quantities in the viscoelastic body. Instead, they are the pseudovariables. The pseudostrain $\varepsilon_{k l}^{R}$ is defined by Eq. (2):

$$
\varepsilon_{k l}^{R}=\frac{1}{E_{R}} \int_{0}^{t} E_{i j k l}(t-\tau) \frac{\partial \varepsilon_{k l}}{\partial \tau} d \tau,
$$

where $E_{R}$ is a reference modulus. According to Eq. (2), Eq. (1) can be rewritten as

$$
\sigma_{i j}=E_{R} \varepsilon_{k l}^{R}
$$

which revealed the linear relationship between the stress and pseudostrain.

\section{Calculation of Pseudostrain Based on Cyclic Loading Test.}

2.1. Relaxation Modulus Determined from the Dynamic Mechanical Test. From the above, we can see that the relationship between stress, strain and time can be obtained. However, to find the pseudostrain, the constitutive model is needed to be obtained, which can characterize the nature of the asphalt mixture. The relaxation modulus of the material can be deduced from the Wiechert model and described in the form of Prony series [11] as shown below:

$$
E(t)=E_{e}+\sum_{n=1}^{N} E_{n} e^{-t / \rho_{n}},
$$

where $E_{e}$ is the long-time equilibrium modulus, and it is the steady state value of modulus $E(t)$ when $t$ approaches infinity, $\rho_{n}=\eta_{n} / E_{n}$ is the relaxation time of the $n$th Maxwell unit, and $N$ is the total number of the Maxwell units.

Based on the Wiechert model, the relaxation modulus coefficient can be determined from the storage modulus. First of all, using the relationship between dynamic modulus and phase angle in dynamic modulus experiment [12], we can get the storage modulus. Then the Fourier transform of the constitutive relation of the Wiechert model is used to transform 
the time domain into the frequency domain, and then the storage modulus can be obtained by elastic-viscoelastic correspondence principle [13], as shown in Eq. (5):

$$
E^{\prime}(\omega)=E_{e}+\sum_{n=1}^{N} \frac{\omega^{2} \rho_{n}^{2} E_{n}}{\omega^{2} \rho_{n}^{2}+1}
$$

The storage modulus at each temperature and its master curve are shown in Fig. 1. Because the direct solution of relaxation time can produce nonlinear problems [14], it is hugely inconvenient. It is necessary to solve the corresponding parameters for a given relaxation time in advance. The relaxation time in this paper is determined according to Eq. (6) and then the remaining parameters in the storage modulus are determined by Levenberg-Marquardt + global optimization. The fitting decision coefficient (DC) is 0.998. The fitting results are shown in Fig. 2, and the corresponding parameters are shown in Table 1. Furthermore, the respective coefficients of the relaxation modulus expressed by the Prony series are also known as

$$
\rho_{n}=k \cdot 10^{(n-c)} \quad(n=1,2,3, \ldots, N),
$$

where $k$ is a constant of 2 , and according to the experimental time range and the requirements of fitting analysis, $N=8$ and $c=5$.

$\mathrm{T}$ a b 1 e 1

The Fitted Parameters in Eq. (5)

\begin{tabular}{|c|c|c|c|c|c|c|c|c|c|c||}
\hline$n$ & 1 & 2 & 3 & 4 & 5 & 6 & 7 & 8 & $E_{e}$ & DC \\
\cline { 1 - 8 }$\rho_{n}$ & $10^{-4}$ & $10^{-3}$ & $10^{-2}$ & $10^{-1}$ & $10^{0}$ & $10^{1}$ & $10^{2}$ & $10^{3}$ & \multirow{2}{*}{109.3} & \multirow{2}{*}{0.998} \\
\hline$E_{n}$ & 4328.5 & 4276.7 & 4184.5 & 3610.4 & 1996.7 & 878.3 & 183.8 & 73.7 & & \\
\hline
\end{tabular}

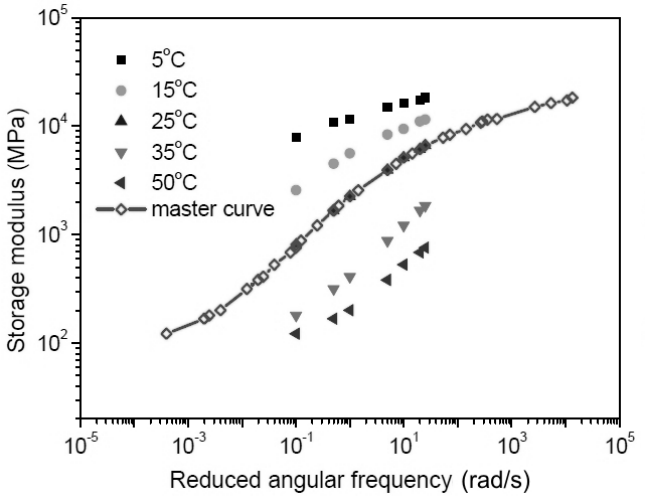

Fig. 1

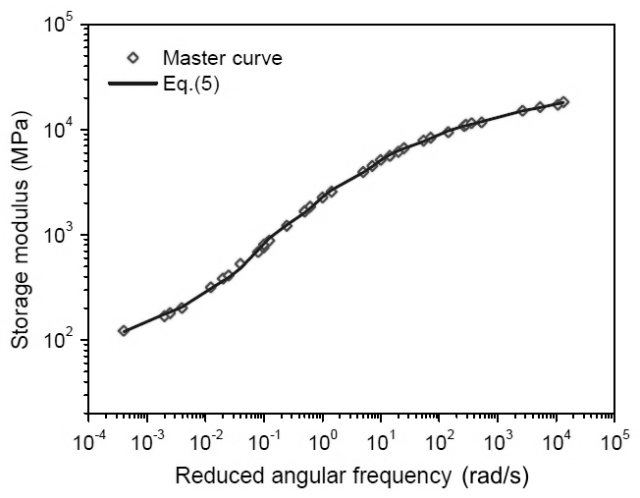

Fig. 2

Fig. 1. Frequency sweep of storage modulus at various temperatures and its master curve.

Fig. 2. Storage modulus master curve and its model fit.

2.2. Stress and Strain Characterization under Cyclic Loading Experiment. In this work, the semi-positive vector cyclic loading test under stress control was conducted on the asphalt mixture specimen with the material testing machine UTM. The stress input $\sigma(t)$ is shown in Fig. 3. The corresponding strain response curve is given in Fig. 4. 
$\mathrm{T}$ a b 1 e 2

Grading of AC-13 Dense-Graded Asphalt Mixture

\begin{tabular}{|c|c|c|c|c|c|c|c|c|c|c||}
\hline \multirow{2}{*}{$\begin{array}{c}\text { Aggregate gradation } \\
\text { sieve sizes (mm) }\end{array}$} & \multicolumn{10}{|c|}{ Percentage passing (\%) } \\
\cline { 2 - 11 } & 16 & 13.2 & 9.5 & 4.75 & 2.36 & 1.18 & 0.6 & 0.3 & 0.15 & 0.075 \\
\hline Sieve grading & 100 & 96.3 & 77.3 & 54.1 & 37.9 & 27.6 & 19.1 & 14.7 & 10.4 & 6.2 \\
\hline
\end{tabular}

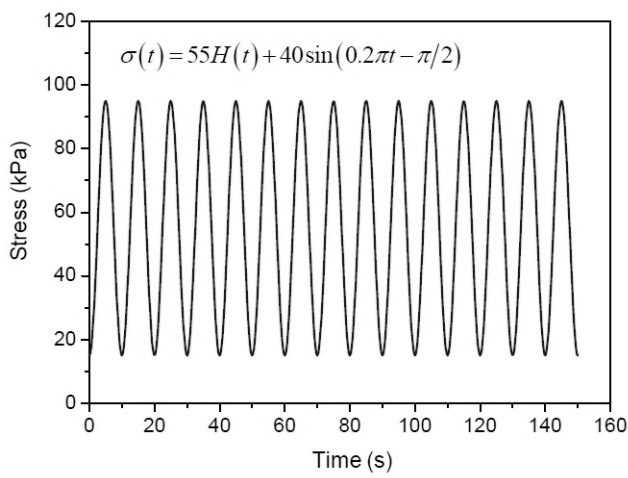

Fig. 3. Stress input in cyclic loading.

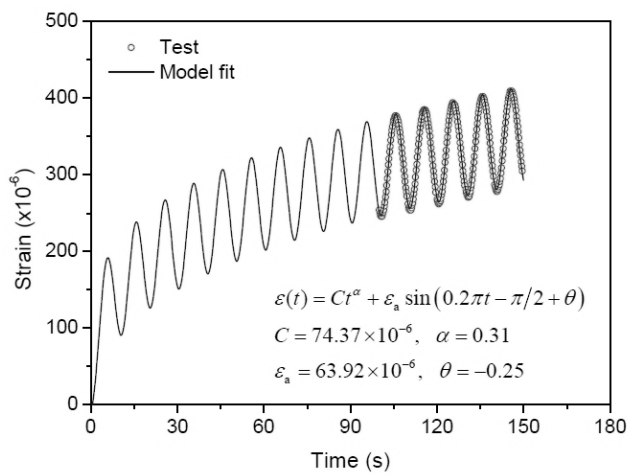

Fig. 4. Strain response and fitting curve.

AC-13 type dense-graded asphalt mixture sample is used in this test [15]. The asphalt used is AH70\# ordinary road asphalt. The ratio of oil to stone is $4.6 \%$. The sample is compacted using a rotary compactor CONTROLS 76-PV2522. The gradation of AC-13 dense-graded asphalt mixture is shown in Table 2.

According to the "highway engineering asphalt and asphalt test procedures" norms [16], the tests were carried out with UTM. A pre-loading of $0.3 \mathrm{kN}$ was conducted for $1 \mathrm{~min}$ to avoid the impact of mechanical clearance. To weaken the end friction, PTFE films were put on the contact surfaces. The experiment was divided into three groups, and all tests were conducted with a loading frequency of $0.1 \mathrm{~Hz}$ and a stress amplitude of $40 \mathrm{kPa}$, the test temperature was controlled at $25^{\circ} \mathrm{C}$. The cyclic loading curve is shown in Fig. 3. The strain response curve is shown in Fig. 4, only revealing the last five cycles.

The loading stress history applied in the test is given below:

$$
\sigma(t)=\sigma_{0} H(t)+\sigma_{a} \sin (0.2 \pi t-\pi / 2)
$$

where $H(t)$ is the Heaviside step function, and $\sigma_{0}=55 \mathrm{kPa}, \sigma_{a}=40 \mathrm{kPa}$.

According to the Boltzmann superposition principle, the total strain response to the applied stress history is decomposed into two parts: the static strain response $\varepsilon_{1}$ and the sinusoidal strain response $\varepsilon_{2}$ :

$$
\left\{\begin{array}{l}
\varepsilon_{1}(t)=C t^{\alpha} \\
\varepsilon_{2}(t)=\varepsilon_{a} \sin (0.2 \pi t-\pi / 2+\theta),
\end{array}\right.
$$

where $C$ and $\alpha$ are model constants, $\varepsilon_{a}$ is the strain amplitude corresponding to the cyclic stress amplitude $\sigma_{a}$, and $\theta$ is the phase angle due to viscoelasticity. Thus, the total strain is given by

$$
\varepsilon(t)=C t^{\alpha}+\varepsilon_{a} \sin (0.2 \pi t-\pi / 2+\theta) .
$$


T a b 1 e 3

Strain Response Fitting Parameters

\begin{tabular}{|c|c|c|c|c||}
\hline$\varepsilon_{0}$ & $\theta, \mathrm{rad}$ & $C$ & $\alpha$ & Decision coefficient \\
\hline $63.92 \cdot 10^{-6}$ & -0.25 & $74.37 \cdot 10^{-6}$ & 0.31 & 0.9979 \\
\hline
\end{tabular}

By combining the Levenberg-Marquardt (LM) method with the global optimization method, the strain response curves were fitted by Eq. (9). The model parameters are obtained and shown in Table 3. The model fitting curve is also plotted in Fig. 4, which is in perfect consistency with the test data in the last five cycles.

2.3. Calculation of Pseudostrain under Cyclic Loading. According to the relationship between stress and time, the relationship between strain and time, the relationship between stress and strain can be obtained as shown in Fig. 5. From the stress-strain curve, it can be seen that the asphalt mixture has apparent hysteretic behavior under cyclic loading, and the curve gradually moves towards the right side with the increases in cycles.

When the pseudostrain is calculated according to the definition of pseudostrain in Eq. (2), the stress vs. strain curve is converted to the stress vs. pseudostrain curve, as shown in Fig. 6. It can be seen from the stress vs. pseudostrain curve that the material's hysteretic behavior disappears, moreover, the stress vs. pseudostrain curve presents a simple linear relationship, which also satisfies the expression of Eq. (3).

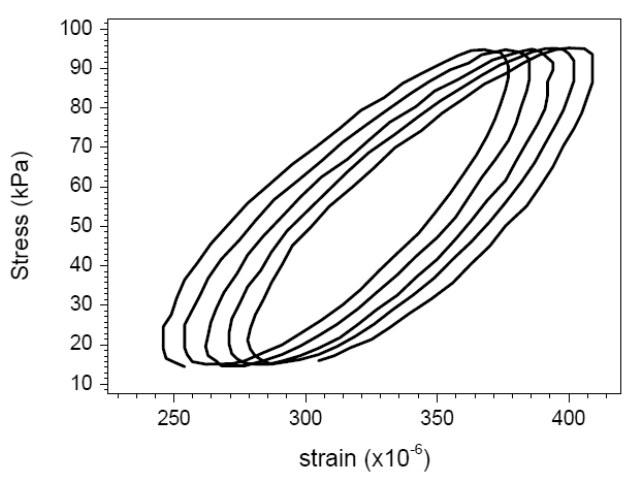

Fig. 5. Stress-strain curve under cyclic loading.

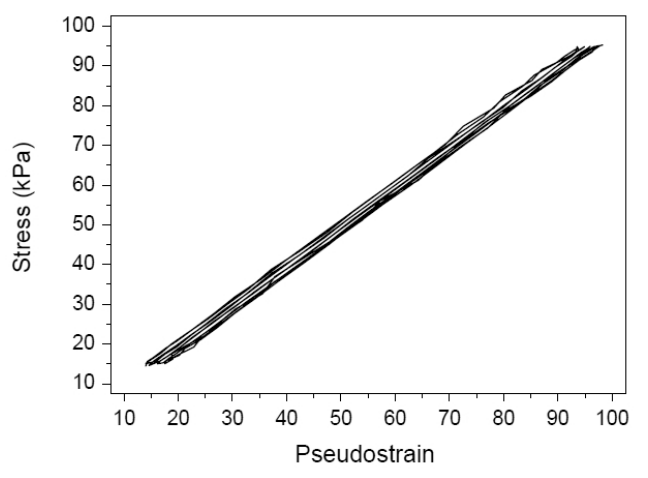

Fig. 6. Stress-pseudostrain curve.

The pseudostiffness, $S^{R}$, is defined by

$$
S^{R}=\frac{d \sigma}{d \varepsilon^{R}} .
$$

\section{Pseudovariables and Fatigue Damage.}

3.1. Pseudostiffness under Cyclic Loading. The above pseudostrain is based on the theory of viscoelasticity and various ideal conditions and assumptions [17]. The effect of heavy cyclic loading on the damage of asphalt mixture is not considered, and the damage of asphalt mixture is the process of microcracks and/or microvoids initiation and expanding during the cyclic loading. Due to the randomness of these microcracks and microvoids, their propagation mechanism is very complicated. Therefore, it is difficult to deduce the fatigue damage law directly through theoretical derivation.

As can be seen from the stress vs. pseudostrain curve in Fig. 6, the pseudostiffness $S^{R}$ is approximately 1 in magnitude, in such case the damage is negligible during the cyclic loading because the maximum stress is less than $100 \mathrm{kPa}$. 
To study the damage evolution in cyclic deformation of asphalt mixtures, the stress-controlled cyclic tests with a stress amplitude of $400 \mathrm{kPa}$ at a frequency of $0.1 \mathrm{~Hz}$ were conducted up to 2000 cycles. The stress vs. strain curves at 1st, 10th, 100th, 1000th and 2000th cycles were recorded and converted into stress vs. pseudostrain curves according to the above method, as shown in Fig. 7. Linear fitting to Eq. (3) with the stress vs. pseudostrain curves, as shown in Fig. 7, provides the pseudostiffness for different load cycles, which is listed in Table 4.

$\mathrm{T}$ a b 1 e 4

The Pseudostiffness at Various Load Cycles

\begin{tabular}{|c|c|c|c|c|c|c||}
\hline \hline Load cycle & 0 cycle & 1 st cycle & 10 th cycle & 100 th cycle & 1000 th cycle & 2000th cycle \\
\hline$S^{R}$ & 1 & 0.9817 & 0.9323 & 0.8791 & 0.7927 & 0.7086 \\
\hline
\end{tabular}

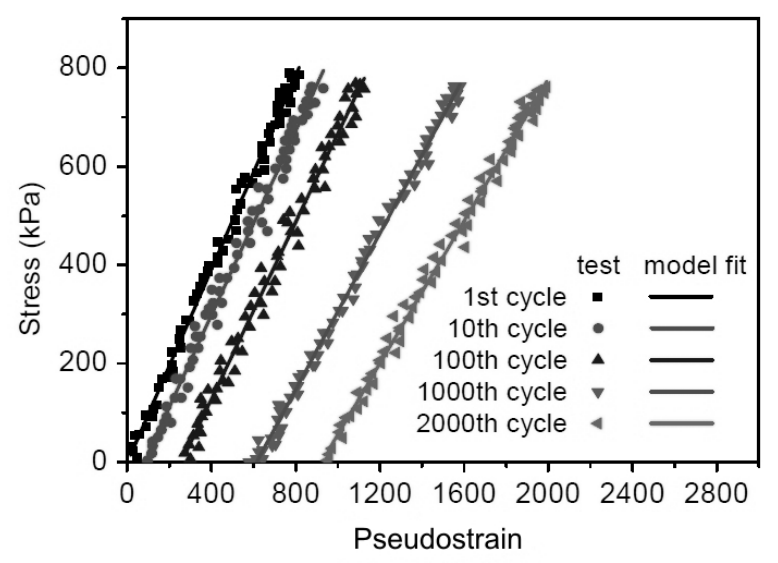

Fig. 7. Stress vs. pseudostrain curves: test and model fit.

3.2. Fatigue Damage Model Based on Pseudorigidity. The damage in the material should be divided into two parts [18] corresponding to static damage and fatigue damage respectively. According to phenomenological law, the static damage variable $D_{s}$ is established as below:

$$
D_{s}=D_{s \infty}\left(1-e^{b N}\right)
$$

where $D_{s \infty}$ and $b$ are material parameters depending on the loading stress amplitude, frequency, and temperature.

The fatigue damage $D_{f}$ can be established according to Miner damage model, as shown in Eq. (12):

$$
D_{f}=\frac{N}{N_{f}},
$$

where $N_{f}$ is the ideal fatigue life and $N$ is the load cycles.

The ideal fatigue life is the cycles up to failure under the cyclic load of the perfect state. However, the process from fatigue damage to fatigue failure is the process of energy release. The process is concise and is affected by the experimental conditions, operational errors and the anisotropy between samples in each group. As a result, the actual fatigue life of each specimen does not reach the ideal fatigue life. Thus the establishment of the relationship between the real fatigue life and the perfect fatigue life is shown in Eq. (13): 


$$
N_{f}=m N_{r f}
$$

where $m=1.1$ is the compensation factor and $N_{r f}$ is the actual fatigue life. Thus the total damage $D$ can be obtained by summing the fatigue damage and the static damage as below:

$$
D(N)=D_{s}(N)+D_{f}(N)=D_{s \infty}\left(1-e^{-b N}\right)+\frac{N}{1.1 N_{r f}}, \quad 0 \leq N \leq N_{r f} .
$$

It is evident from Table 4 that the pseudostiffness decreases with the increase in load cycles. We define the damage variable $D$ by the change of the pseudostiffness as below:

$$
D=\frac{S_{0}^{R}-S^{R}}{S_{0}^{R}},
$$

where $S^{R}$ is the pseudostiffness at any load cycle and $S_{0}^{R}$ is the initial pseudostiffness. It is seen from Table 4 that $S_{0}^{R}=1$. Using Eq. (15) we can calculate the damage degree for different load cycles, and the calculation results are shown in Fig. 8.

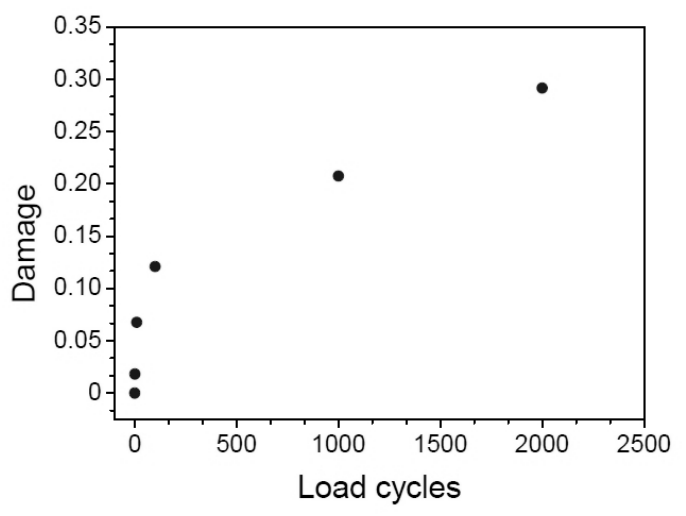

Fig. 8. Observed damage variation with load cycles.

3.3. Model Verification and Fatigue Damage Analysis. By use of the LevenbergMarquardt (LM) method and generalized global optimization method, the damage for 1, 10, 100, and 1000 cycles in Fig. 8 were fitted with Eq. (14), and the parameters in Eq. (14) are determined to be that $D_{s \infty}=0.1070, b=0.0907$, and $N_{r f}=9441$. Therefore, the damage evolution equation can be described by Eq. (16):

$$
D(N)=0.10701\left(1-e^{-0.0907 N}\right)+\frac{N}{10385}
$$

It is clear that Eq. (16) satisfies the initial condition, in which case $D(0)=0$. Moreover, the sample will be damaged to fracture when it is loaded by $N_{r f}$ cycles. According to Eq. (16), $D\left(N_{r f}\right)=D(9441)=1.01$, which meet the failure criterion.

The damage at the 2000th cycle is not used in the model fitting of Eq. (16). To check the validity of the model, the damage in the 2000th cycle is predicted using Eq. (16), $D(2000)=0.2996$, while the corresponding observed damage in Fig. 8 is 0.2914. The prediction is in good agreement with the test, as shown in Fig. 9. 


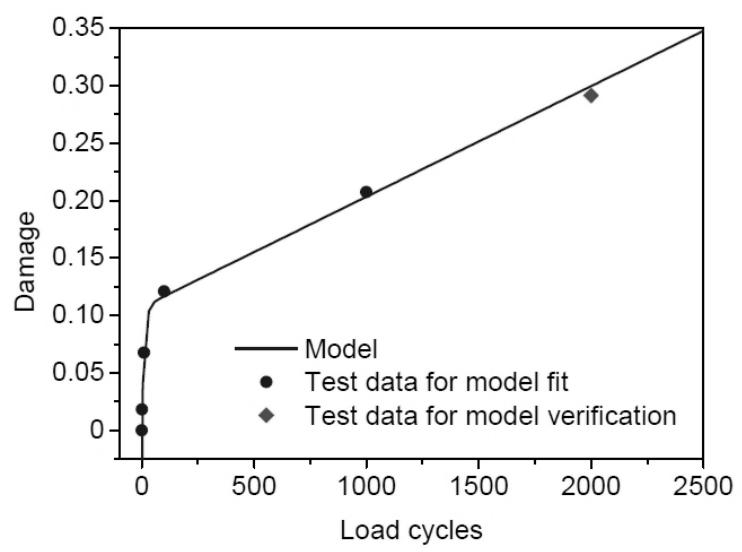

Fig. 9. Damage model prediction vs. test data.

\section{Conclusions}

1. Based on the Schapery pseudovariable theory and Wiechert model, the experimental stress vs. strain curves of asphalt mixture under semi-positive vector cyclic loading are transformed into stress vs. pseudostrain curves. The stress vs. pseudostrain curve eliminates the hysteresis effect and load history dependence and presents a simple linear relationship.

2. The slope of the stress vs. pseudostrain curve gradually decreases with the increase of fatigue cycles. The damage variable is defined by the change of the pseudostiffness. Meanwhile, a damage evolution equation is constructed by considering the superposition of the static damage and the fatigue damage. The prediction by the proposed damage model is in good agreement with the test data.

Acknowledgments. This work was supported by the National Natural Science Foundation of China (11572275), the Key Laboratory of Road Structure and Material of Ministry of Transportation (Beijing) and the Hunan Provincial Innovation Foundation for Postgraduate, China (CX2018B074).

1. M. R. Kakar, M. O. Hamzah, and J. Valentin, "A review on moisture damages of hot and warm mix asphalt and related investigations," J. Clean. Prod., 99, 39-58 (2015).

2. H. J. Lee, J. S. Daniel, and Y. R. Kim, "Continuum damage mechanics-based fatigue model of asphalt concrete," J. Mater. Civil Eng., 12, No. 2, 105-112 (2000).

3. Y. R. Kim, D. H. Allen, and D. N. Little, "Computational model to predict fatigue damage behavior of asphalt mixtures under cyclic loading," Transport. Res. Rec. J. Transport. Res. Board, 1970, No. 1, 196-206 (2006).

4. Q. Li, H. J. Lee, and T. W. Kim, "A simple fatigue performance model of asphalt mixtures based on fracture energy," Constr. Build. Mater., 27, No. 1, 605-611 (2012).

5. H. X. Guan, J. L. Zheng, and Q. S. Zhang, "Viscoelastic fatigue damage model of bituminous mixture," Mech. Eng., 29, No. 2, 50-53 (2007).

6. H. Z. Zhu, H. Yan, and B. M. Tang, "Damage model of interaction between fatigue and creep for asphalt mixture," China J. Highway Transport, 24, No. 4, 15-20 (2011). (in Chinese)

7. Y. R. Kim, Modeling of Asphalt Concrete, ASCE Press (2008), p. 164.

8. F. Safaei, C. Castorena, and Y. R. Kim, "Linking asphalt binder fatigue to asphalt mixture fatigue performance using viscoelastic continuum damage modeling," Mech. Time-Depend. Mat., 20, No. 3, 1-25 (2016). 
9. R. M. Christensen, Theory of Viscoelasticity, Academic Press, New York (1982).

10. R. A. Schapery, "Correspondence principles and a generalized $J$ integral for large deformation and fracture analysis of viscoelastic media," Int. J. Fracture, 25, No. 3, 195-223 (1984).

11. F. M. Liu and D. Y. Wang, "Multiscale simulation of damage evolution of asphalt mixtures," China J. Highway Transport, 23, No. 2, 1-6 (2010). (in Chinese)

12. Y. Q. Zhao, J. M. Tang, and L Bai, "Determination of relaxation modulus using complex modulus of asphalt mixture," J. Build. Mater., 15, No. 4, 498-502 (2010).

13. W. Cao, A. Norouzi, and Y. R. Kim, "Application of viscoelastic continuum damage approach to predict fatigue performance of BinZhou perpetual pavements," J. Traffic Transport. Eng., 3, No. 2, 104-115 (2016). (in Chinese)

14. J. K. Chen, Z. P. Huang, H. J. Chu, and S. L. Bai, "Nonlinear viscoelastic constitutive relations based on the rate sensitive relaxation time under the condition of uniaxial stress," Acta Polym. Sin., 138, No. 3, 415-417 (2003).

15. G. M. Chen, Y. Q. Tan, Z. R. Wang, and Y. W. Zhang, "Fractal study of grading curve trend of the asphalt mixtures," J. Highway Transport. Res. Dev., 22, No. 1, 2-4 (2005).

16. JTG E20-2011. Standard Test Methods of Bitumen and Bituminous Mixtures for Highway Engineering [in Chinese], China Communication Press, Beijing (2000).

17. H. D. Benedetto, C. D. L. Roche, and H Baaj, "Fatigue of bituminous mixtures," Mater. Struct., 37, No. 3, 202-216 (2004).

18. J. L. Zheng and S. T. Lv, "Nonlinear fatigue damage model for asphalt mixtures," China J. Highway Transport, 22, No. 5, 21-28 (2009). (in Chinese) 